Cathodoluminescence and photoluminescence study of plastically deformed ZnTe bulk single crystals

This content has been downloaded from IOPscience. Please scroll down to see the full text. 2001 Semicond. Sci. Technol. 16289

(http://iopscience.iop.org/0268-1242/16/5/302)

View the table of contents for this issue, or go to the journal homepage for more

Download details:

IP Address: 147.96.14.16

This content was downloaded on 11/10/2013 at 17:58

Please note that terms and conditions apply. 


\title{
Cathodoluminescence and photoluminescence study of plastically deformed ZnTe bulk single crystals $\uparrow$
}

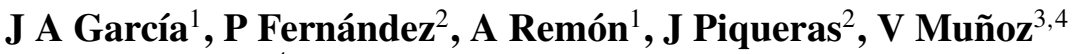 \\ and $\mathbf{R}$ Triboulet ${ }^{4}$ \\ ${ }^{1}$ Departamento de Física Aplicada II, Facultad de Ciencias, Universidad del País Vasco, \\ Lejona, Vizcaya, Spain \\ ${ }^{2}$ Departamento de Física de Materiales, Facultad de Físicas, Universidad Complutense, \\ 28040 Madrid, Spain \\ ${ }^{3}$ Departamento de Física Aplicada and Instituto de Ciencia de Materiales de la Universidad \\ de Valencia (ICMUV), c/Doctor Moliner no 50, E-46100 Burjassot (Valencia), Spain \\ ${ }^{4}$ Laboratoire de Physique des Solides de Bellevue, Centre National de la Recherche \\ Scientifique, (LPSB/CNRS), 1 Place Aristide Briand, F-92195 Meudon, France
}

Received 9 November 2000, accepted for publication 19 January 2001

\begin{abstract}
Samples of zinc telluride bulk single crystals, which were deformed in uniaxial compression, have been studied by photoluminescence (PL) and cathodoluminescence (CL). As a particular feature the deformed samples present a PL emission band peaked at $603 \mathrm{~nm}$, whose intensity increases as the plastic deformation does. This band is related to the density of dislocations produced during the interaction of slip systems. This hypothesis is supported by CL images, which reveal the activation of the successive slip systems corresponding to different levels of deformation.
\end{abstract}

\section{Introduction}

Zinc telluride (ZnTe) is a very promising material in the field of optoelectronics. However, to date two main problems still constitute a strong handicap to the extensive technological application of this material. In the as-grown state, ZnTe shows only p-type conductivity, while n-type conductivity is difficult to achieve. The strong self-compensation mechanisms that are mainly responsible for this behaviour are not fully understood but it is widely accepted that the presence of defects, which introduce localized electronic levels in the bandgap, may play an important role $[1,2]$. On the other hand, structural defects diminish the carrier lifetime and the potential usefulness for commercial applications [3]. In particular, dislocations influence the electronic properties of crystals, because they introduce new energy levels and produce electric and elastic strain fields, which shift the already existing levels.

Much work has been currently devoted to the growth and characterization of ZnTe-based structures, most of which have been grown on GaAs substrates [4]. However, the lattice mismatch and the difference in thermal expansion coefficients between substrate and epilayer are a source of

$\dagger$ This paper is dedicated to the memory of Dr A Remón, who passed away last August. strain and structural defects with a detrimental effect on the properties of the heterostructures. The natural way to avoid these problems would be to grow the structures on ZnTe substrates. Accordingly a new research effort has been promoted to improve the growth processes of bulk $\mathrm{ZnTe}$ and to overcome the problems related to structural imperfection and incorporation of residual impurities.

On the other hand there are a considerable number of publications dealing with photoluminescence (PL) studies on ZnTe layers grown on different substrates, but the bulk crystals have been less analysed, especially in the bandgap energy region. Emission peaks related to extended structural defects have been observed in ZnTe crystals grown on different substrates $[5,6]$. The origin of these peaks has been ascribed to the dislocation network due to the mismatch at the interfaces, but the mechanism of these processes and the defects involved have not yet been determined.

A previous PL work [7] on deformed bulk single crystals of $\mathrm{ZnTe}$ shows that deformation dramatically increases the number of non-radiative centres in the crystal. The deformed samples present different deep-level-related emission bands and a decrease of the overall PL intensity. In this paper we extend our previous study [7] to investigate the defect structure of deformed single crystals of bulk $\mathrm{ZnTe}$ by comparing 
the results obtained by cathodoluminescence (CL) in the scanning electron microscope and PL experiments. Both techniques provide very valuable information on deep-levelrelated transitions. Particularly the attainable spatial resolution with the CL measurements will be very useful to investigate the distribution of the defects involved in the different emissions.

\section{Experimental method}

The experiments were performed on nominally undoped ZnTe crystals grown by the cold travelling heater method CTHM (see [8] and [9] for details). The samples were cut from the ingots with a wire saw, ground with abrasive powder, polished with diamond paste, chemically polished with a $5 \%$ Br:methanol solution and finally washed in deionized water. Single-crystal samples with typical dimensions of about $6 \times 4 \times 1.5 \mathrm{~mm}^{3}$ were prepared.

Samples were uniaxially compressed at room temperature at strains ranging from 1 to $8 \%$, using an Instron machine model 4467 at a fixed strain rate of $2 \times 10^{-5} \mathrm{~s}^{-1}$. In some cases, to maximize the effect of axial compression, the $\{111\}$ slip plane was tilted by $45^{\circ}$ from the compression axis.

To better compare PL band emission intensities from samples with different degrees of deformation, the experimental conditions should be kept constant; however, sometimes this is difficult to achieve if the samples must be removed from the cryostat of the spectrometer to perform successive deformation steps. To avoid this problem, in this paper, the samples were wedge shaped, so that their dimension perpendicular to the compression faces varied from 2.7 to $2.9 \mathrm{~mm}$; the compression faces were $8 \times 1.5 \mathrm{~mm}^{2}$ in size. This geometrical configuration allows creation of a strain gradient, and it permits the luminescence measurements to be performed in identical conditions for different deformation degrees. The deformation degree of the sample was monitored by observing the optical anisotropy generated (photo-elastic effect) in a polarization microscope, and its value was estimated by comparison with the results obtained from other single crystals in which the deformation degree had been previously determined. In this way the deformation along the axis was estimated to be between 0 and about $8 \%$.

PL measurements were performed in a CD900 spectrometer system from Edinburgh Instruments, with a R955 photomultiplier in a Peltier cooled housing. The samples were cooled in the 10-300 $\mathrm{K}$ temperature range in a closed helium cryostat and were excited by the $514 \mathrm{~nm}$ line of an $\mathrm{Ar}^{+}$laser using the laser port of the CD900 spectrometer. The laser intensity was $20 \mathrm{~mW}$ and the spot size was $0.6 \mathrm{~mm}$ in diameter.

CL measurements were performed in a Hitachi S-2500 scanning electron microscope at beam energy of $25 \mathrm{keV}$ and temperatures between 80 and $300 \mathrm{~K}$. The experimental set-up for CL measurements using a Hamamatsu R928 photomultiplier has been previously described [10].

\section{Results and discussion}

Figure 1 shows the near-band-edge PL emission from different points of the sample. In this plot, curve a corresponds to the undeformed (or slightly deformed) region and $\mathrm{b}$ and $\mathrm{c}$ to increasingly strained regions.

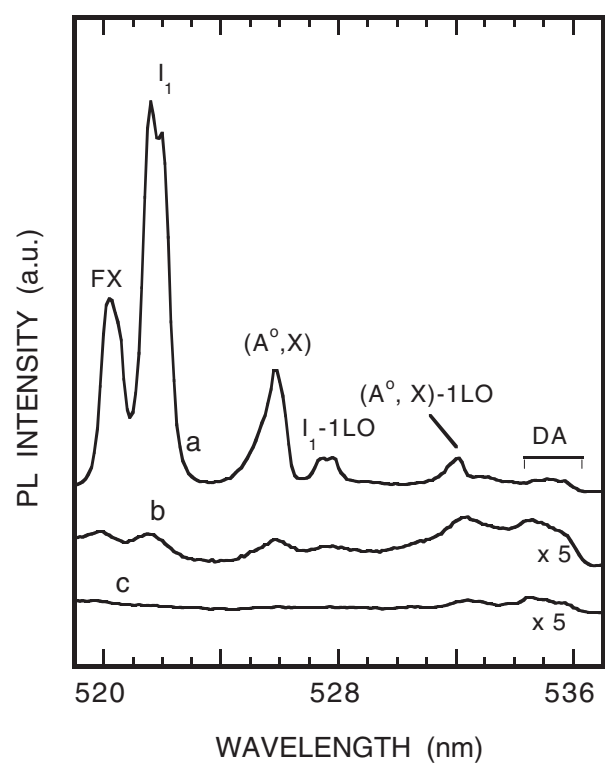

Figure 1. Emission spectra in the excitonic zone, at $10 \mathrm{~K}$ and $514 \mathrm{~nm}$ excitation, of a gradient-deformed crystal: a, in the less deformed end of the sample; b, $4 \%$, and c, $8 \%$ deformation.

The spectrum from undeformed areas shows three emission bands peaked at 520.5, 521.5 and $525.7 \mathrm{~nm}$. The first one corresponds to free exciton recombination (FX) and the other two have been associated with bound exciton transitions. The dominant emission is the $I_{1}$ line centred at $521.5 \mathrm{~nm}$, which has been attributed to the recombination of an exciton bound to a neutral shallow acceptor, related to $\mathrm{Cu}$ impurities $[7,9]$. The line at $525.7 \mathrm{~nm}$ (labelled $\left(A^{o}, X\right)$ in the figure), has been also ascribed to an exciton bound to acceptor transition: the involved acceptor could be either a single zinc vacancy or a complex formed between the $V_{\mathrm{Zn}}$ and a donor [7,9]. Also, the $I_{1}$ and $\left(A^{o}, X\right)$ phonon replicas can be seen. In the range 534$536 \mathrm{~nm}$ can be observed residual free to bound $\left(e, A^{o}\right)$ and DA emissions. As can be observed in the two other spectra of figure 1, plastic deformation inhibits, almost completely, the near-band-edge emissions. Deformation at points where spectra $b$ and $c$ were recorded was estimated to be 4 and $8 \%$ respectively. For $8 \%$ plastic deformation only a very lowintensity, almost structureless spectrum remains.

In contrast to the above described behaviour for the intensity of the near-band-edge emissions, the intensity of the deep-level-related emissions increases with deformation. This effect can be clearly appreciated in the spectra shown in figure 2. For simplicity, only the results of three different positions of the sample are reported in this figure, that has been labelled according to the deformation degree: a, low strain; $\mathrm{b}$, around $4 \%$, and $\mathrm{c}$, around $8 \%$. For the slightly deformed zone a the deep-level emission is much less intense than the exciton-related one. It shows the $\mathrm{DA}_{\mathrm{Cu}}$ band at $552 \mathrm{~nm}$, which involves $\mathrm{Cu}$ as an acceptor, and its LO replicas (see [7,9]). In this figure the $Y_{2}$ peak at $575 \mathrm{~nm}$, which has been related to structural defects in epitaxial layers $[5,6]$, is clearly observed. Also a weak $Y_{1}$ peak $(567 \mathrm{~nm})$ at the position of the $\mathrm{DA}_{\mathrm{Cu}}$ phonon replica appeared. Finally, the red emission at $660 \mathrm{~nm}$ that has been related to oxygen centres [7,9], is also present.

On the other hand, plastic deformation decreases the $\mathrm{DA}_{\mathrm{Cu}}$ band and increases the $Y_{1}$ and $Y_{2}$ peak intensities. Also an 


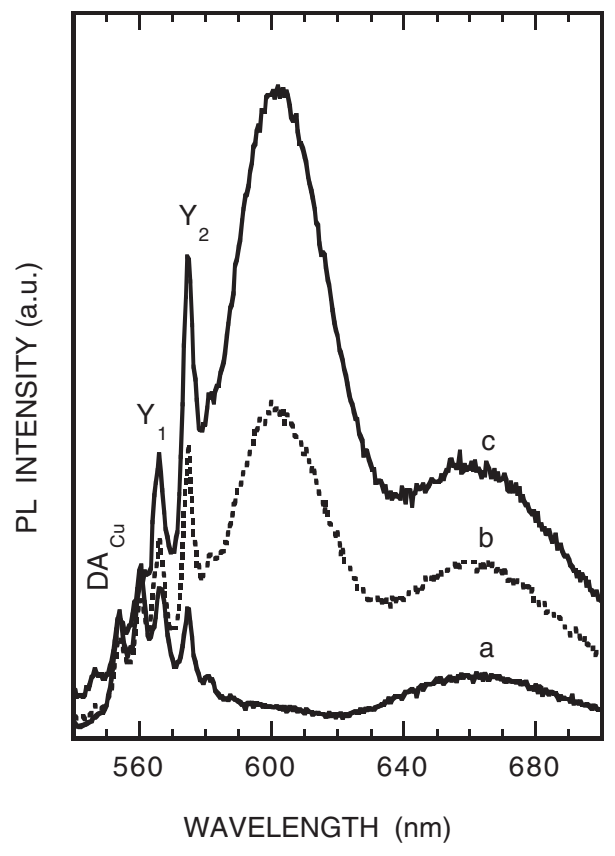

Figure 2. PL spectra in the deep-level region of the same sample and at the same deformation zones: $\mathrm{a}-\mathrm{c}$ as indicated in figure 1 .

emission band centred at $603 \mathrm{~nm}$ appears in the most severely damaged regions and even becomes the dominant feature for the region with $8 \%$ deformation, as observed in curves $b$ and $c$.

CL spectra are consistent with the above results and do not reveal additional features. As in the case of PL nearband-edge emission decreases as deformation increases, while deep-level emissions show the opposite behaviour. Also an increase of the dislocation-related emission is observed in the CL spectra from the most severely deformed areas. However, as will be described, valuable information can be obtained from the spatial distribution of the luminescence as observed in the CL images. If we compare the CL and PL results obtained in the near-band-edge zone, it can be seen that the PL as well as CL emission intensity decreases considerably as the degree of plastic deformation introduced into the sample increases. This can be explained taking into account that deformation creates a high number of defects, many of which could be involved in a variety of non-radiative transitions, able to compete with the radiative ones either already present in the sample, or related to deformation-induced defects. On the other hand, as many of the defects produced during deformation can introduce deep levels an increase of the corresponding emissions should be expected.

Typical CL images, which were recorded in deformed areas, are shown in figure 3. Panchromatic images of deformed samples present a pattern of alternate dark and bright lines. Since the emission from deformed regions is much weaker, the dark lines would indeed correspond to slip lines. In the regions with low deformation degree only one slip system is clearly observed (figure 3(a)); however, up to three different slip systems show up in the highly deformed regions (figure $3(b)$ ). As reported in a previous work [8], images from undeformed regions do not show these dark line patterns.

PL results for these areas show a clear increase of the dominant $603 \mathrm{~nm}$ emission. The relative intensity of this band

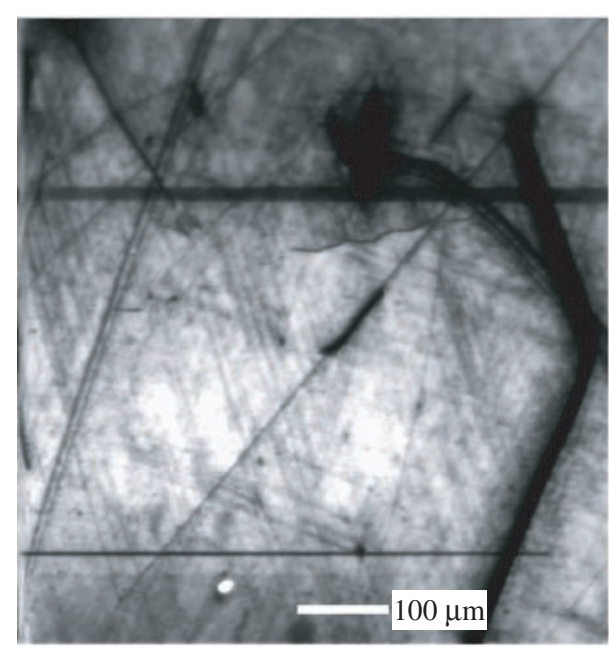

(a)

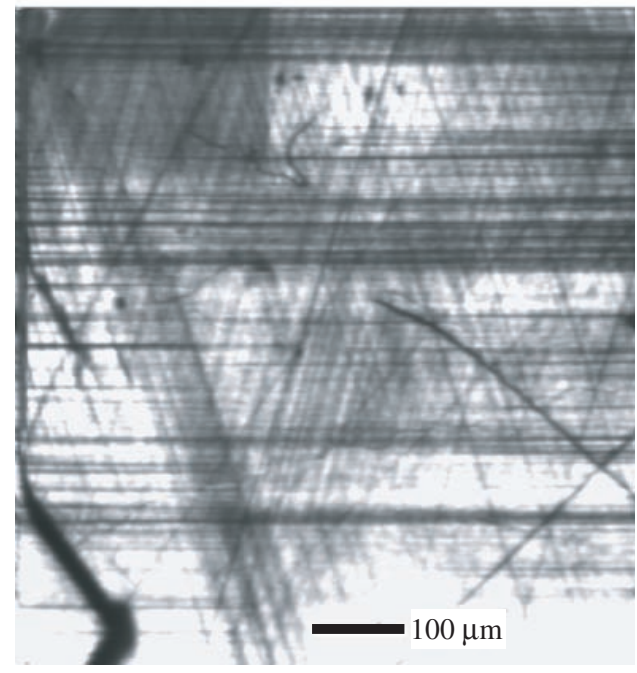

(b)

Figure 3. Panchromatic CL images from (a) a low-deformation region and $(b)$ a high-deformation region.

seems to increase as the number of slip lines does, and this can be directly related to the fact that it can be directly related to a high density of dislocation, like that produced during the interaction of slip systems.

The above assumption can be reinforced by the fact that this band has also been detected in undeformed polycrystals but never in single crystals [7], before deformation, so its origin can be attributed to the strongly dislocated regions, for instance the grain boundaries in polycrystals where a pile-up of dislocations occurs.

\section{Conclusions}

The $603 \mathrm{~nm}$ PL emission band, which appears in deformed samples, is related to the high density of dislocations produced during the interaction of slip systems. This hypothesis is supported by the increase in intensity of the band as the plastic deformation increases and by the CL images, which reveal the activation of the successive slip systems corresponding to the different levels of deformation. 


\section{Acknowledgments}

This paper was partially supported by the Spanish Comisión Interministerial para Ciencia y Tecnología under grants MAT98-0975-C02-01 and 1FD97-0086.

\section{References}

[1] Marfaing Y 1996 J. Cryst. Growth 161205

[2] Pautrat J L, Magnea N and Faurie J P 1982 J. Appl. Phys. 53 8668

[3] Nakamura S 1997 MRS Bull. 2229
[4] Kuhn W S et al 1995 Prog. Cryst. Growth Charac. Mater. 31 119

[5] Naumov A, Wolf K, Reisinger T, Stanzl H, Wagner H P and Gebhardt W 1993 Physica B 185250

[6] Wolf K, Naumov A, Reisinger T, Kastner M, Stanzl H, Kuhn W and Gebhardt K W 1994 J. Cryst. Growth 135113

[7] García J A, Remón A, Muñoz V and Triboulet R 1999 J. Cryst. Growth 197794

[8] Fernandez P, García A, Remón A, Piqueras J, Muñoz V and Triboulet R 1998 Semicond. Sci. Technol. 13410

[9] García J A, Remon A, Muñoz V and Triboulet R 1998 J. Cryst. Growth 191685

[10] Domínguez-Adame F, Piqueras J and Fernández P 1991 Appl. Phys. Lett. 58257 